\title{
TOPOLOGICAL CLASSIFICATION AND FINITE DETERMINACY OF KNOTTED MAPS
}

\author{
JUAN J. NUÑO-BALLESTEROS AND RODRIGO MENDES
}

\begin{abstract}
We show that the knot type of the link of a real analytic map germ with isolated singularity $f:\left(\mathbb{R}^{2}, 0\right) \rightarrow\left(\mathbb{R}^{4}, 0\right)$ is a complete invariant for $C^{0}$ - $\mathscr{A}$-equivalence. Moreover, we also prove that isolated instability implies $C^{0}$-finite determinacy, giving an explicit estimate for its degree. For the general case of real analytic map germs, $f:\left(\mathbb{R}^{n}, 0\right) \rightarrow\left(\mathbb{R}^{p}, 0\right)(n \leq p)$, we use the Lojasiewicz exponent associated to the Mond's double point ideal $I^{2}(f)$ to obtain some criteria of Lipschitz and analytic regularity.
\end{abstract}

\section{INTRODUCTION}

In a previous paper [13], we consider analytic map germs $f:\left(\mathbb{R}^{2}, 0\right) \rightarrow\left(\mathbb{R}^{4}, 0\right)$ with isolated singularity. This means that there exists a representative $f: \mathcal{U} \rightarrow \mathcal{V}$ such that $f$ is a topological embedding on $\mathcal{U}$ and an immersion on $\mathcal{U} \backslash\{0\}$. In particular, its image $X=f(\mathcal{U})$ is a surface with isolated singularity surface at the origin in $\mathbb{R}^{4}$. By the cone structure theorem, the topological type of the germ $f$ is determined by the knot type of its link $K(f)=X \cap \mathbb{S}_{\epsilon}^{3}$ (where $\epsilon>0$ is a Milnor-Fukuda radius of $f$ ). In the first part of this paper, we show the converse of this, namely, that the knot type of the link $K(f)$ is a topological invariant.

In the second part of the paper, we consider the general case of analytic map germs $f:\left(\mathbb{R}^{n}, 0\right) \rightarrow\left(\mathbb{R}^{p}, 0\right)$ with isolated singularity $(n \leq p)$. We define a pair of invariants which are Lojasiewicz exponents associated to the Mond ideal $I^{2}(f)$. Recall that there exists a $p \times n$-matrix $\alpha$ with entries in $\mathscr{E}_{2 n}$ such that

$$
f\left(x^{\prime}\right)-f(x)=\alpha\left(x, x^{\prime}\right)\left(x^{\prime}-x\right),
$$

and that the ideal $I^{2}(f) \subset \mathscr{E}_{2 n}$ generated by the functions $f_{i}(y)-f_{i}(x)$, with $i=1, \ldots, p$, and by the $n \times n$-minors of $\alpha$ (here $\mathscr{E}_{2 n}$ is the local ring of analytic function germs from $\left(\mathbb{R}^{n} \times \mathbb{R}^{n}, 0\right)$ to $\left.\mathbb{R}\right)$.

The first invariant $\mathcal{L}_{0}(\tilde{\Delta} f)$ is called the isolated singularity exponent and is defined as the Lojasiewicz exponent of $I^{2}(f)$ with respect to the maximal ideal $\mathcal{M}_{2 n}$. It has the property that $\mathcal{L}_{0}(\tilde{\Delta} f)<\infty$ if and only if $f$ has isolated singularity. We use this invariant to prove that if $f:\left(\mathbb{R}^{2}, 0\right) \rightarrow\left(\mathbb{R}^{4}, 0\right)$ has isolated singularity, then $f$ is $C^{0}$-finitely determined. In fact, we give an explicit estimate for the degree of $C^{0}$-determinacy. It is

2010 Mathematics Subject Classification. 58K15; 14P25, 57M25.

Key words and phrases. Lojasiewicz exponent, knots, isolated singularity.

The first author has been partially supported by DGICYT Grant MTM2015-64013-P. 
well known that any finitely determined germ has isolated instability (and in particular, isolated singularity when $n=2$ and $p=4$ ), by the Mather-Gaffney criterion (see [21]). But the converse is not true in the real analytic case. A natural open question is if isolated instability implies $C^{0}$-finite determinacy. This is known to be true for function germs (see [12]) and here we answer this question in the case $n=2$ and $p=4$. For the general case of $C^{\infty}$ map germs $f:\left(\mathbb{R}^{n}, 0\right) \rightarrow\left(\mathbb{R}^{p}, 0\right)$, we refer to [5], where they give a sufficient condition for $C^{0}$-finite determinacy in terms of some Lojasiewicz inequalities in the jet space.

The second invariant $\mathcal{L}_{0}(\Delta f)$ is called the double point exponent and is defined as the Lojasiewicz exponent of the ideal generated by $f_{i}(y)-f_{i}(x), i=1, \ldots, p$, with respect to the ideal generated by $x_{j}^{\prime}-x_{j}, j=1, \ldots, n$. In this case, we have that $\mathcal{L}_{0}(\Delta f)<\infty$ if and only if $f$ is injective. We show that this a bi-Lipschitz invariant of $f$ and we use it to prove that $f$ is a bi-Lipschitz embedding if and only if it is a smooth embedding. This result could be seen as a weaker real version of a theorem by Birbrair, Lê, Fernandes and Sampaio [1], where they show that if a complex algebraic set $X \subset \mathbb{C}^{n}$ is Lipschitz regular at a point $x_{0} \in X$, then $X$ is smooth at $x_{0}$.

\section{INVERTIBLE COBORDISMS OF KNOTS}

In this section, we show that two knots which are invertible cobordant from both ends are equivalent. We first recall the notion of peripheral structure of a knot. Along all the paper, a knot $K \subset \mathbb{S}^{3}=\left\{x \in \mathbb{R}^{4} ;\|x\|=1\right\}$ is always a tame knot, unless otherwise stated. Two knots $K_{1}, K_{2}$ are said equivalent if there exist a homeomorphism $\phi: \mathbb{S}^{3} \rightarrow \mathbb{S}^{3}$ such that $\phi\left(K_{1}\right)=K_{2}$.

Definition 2.1. Let $K \subset \mathbb{S}^{3}$ be a knot. Consider $N(K) \subset \mathbb{S}^{3}$ a tubular neighbourhood of $K$, so $N(K)$ is a smooth submanifold homeomorphic to $K \times \mathbb{B}_{2}$. A meridian of $K$ is a simple closed curve $m$ contained in $\partial N(K)$ such that $m$ is not homotopically trivial in $\partial N(K)$, but is homologically trivial in $N(K)$. Analogously, a longitude of $K$ is a simple closed curve $l$ contained in $\partial N(K)$ such that $l$ is homologous to $K$ in $N(K)$. We say that $(m, l)$ is a meridian-longitude pair of $K$.

Definition 2.2. Let $K_{1}, K_{2} \subset \mathbb{S}^{3}$ be two knots and consider a meridian-longitude pair $\left(m_{i}, l_{i}\right)$ of each $K_{i}$ contained in $\partial N\left(K_{i}\right), i=1,2$. Let $\left\langle\left[m_{i}\right],\left[l_{i}\right]\right\rangle$ be the subgroup generated by their classes in $G_{i}=\pi_{1}\left(\mathbb{S}^{3} \backslash N\left(K_{i}\right)\right)$. Suppose that there is an isomorphism $\varphi_{*}$ : $G_{1} \rightarrow G_{2}$. We say that the isomorphism $\varphi_{*}$ preserves the peripheral structure when $\varphi_{*}\left(\left\langle\left[m_{1}\right],\left[l_{1}\right]\right\rangle\right)$ is conjugate to a subgroup of $\left\langle\left[m_{2}\right],\left[l_{2}\right]\right\rangle$ in $G_{2}$.

The following result is a consequence of the works of Dehn [15], Waldhausen [20] and Gordon and Luecke [9]. It means that the knot group plus the peripheral structure information provide a complete invariant of the knot. 
Theorem 2.3. Let $K_{1}, K_{2} \subset \mathbb{S}^{3}$ be two knots such that their knot groups $G_{1}$ and $G_{2}$ are isomorphic. If the isomorphism $\varphi_{*}: G_{1} \rightarrow G_{2}$ preserves the peripheral structure then $K_{1}$ and $K_{2}$ are equivalent.

Proof. If one of the knots is trivial, then $G_{1} \cong G_{2} \cong \mathbb{Z}$ and by Dehn's Lemma [15, the other knot is also trivial. Thus, we can assume that $K_{1}$ and $K_{2}$ are not trivial. Again by Dehn's Lemma, we obtain that the 3-manifolds $\mathbb{S}^{3} \backslash N\left(K_{i}\right)$ are sufficiently large, for $i=1,2$. By Waldhausen theorem (see [20, Corollary 6.5]), there exists a homeomorphism between $\mathbb{S}^{3} \backslash \stackrel{\circ}{N}\left(K_{1}\right)$ and $\mathbb{S}^{3} \backslash \stackrel{\circ}{N}\left(K_{2}\right)$, which can be extended to a homeomorphism between $\mathbb{S}^{3} \backslash K_{1}$ and $\mathbb{S}^{3} \backslash K_{2}$. So, the knots $K_{1}$ and $K_{2}$ are equivalent, by Gordon-Luecke theorem [9].

In a previous paper [13], the authors used the notion cobordism of knots associated to two $C^{0}$ - $\mathscr{A}$-equivalent map germs, but here we are interested in an equivalence which is a little bit stronger: the notion of invertible cobordism from both ends. This appears in a more general context in the works of Stallings [18] and Siebenmann [17]. We recall the definition in the case of knots.

Definition 2.4. Two knots $K_{1}, K_{2} \subset \mathbb{S}^{3}$ are called invertible cobordant from end $K_{2}$ if there exist cobordisms $\left(W_{12} ; K_{1}, K_{2}\right)$ and $\left(W_{21} ; K_{2}, K_{1}\right)$ such that $\left(W_{12} \cup W_{21} ; K_{1}, K_{1}\right)$ is homeomorphic to the product cobordism $\left(K_{1} \times[0,1], K_{1}, K_{1}\right)$. In the same way, we can define invertible cobordism from end $K_{1}$.

Proposition 2.5. If two knots $K_{1}, K_{2} \subset \mathbb{S}^{3}$ are invertible cobordant from both ends, then $K_{1}, K_{2}$ are equivalent.

Proof. Let $\left(W ; K_{1}, K_{2}\right)$ be an invertible cobordism from both ends $K_{1}$ and $K_{2}$. By [19, Proposition 2.1], $\mathbb{S}^{3} \times[0,1] \backslash W$ provides an $h$-cobordism between $\mathbb{S}^{3} \backslash K_{1}$ and $\mathbb{S}^{3} \backslash K_{2}$. It means that the inclusions

$$
i_{1}: \mathbb{S}^{3} \backslash K_{1} \rightarrow \mathbb{S}^{3} \times[0,1] \backslash W, \quad i_{2}: \mathbb{S}^{3} \backslash K_{2} \rightarrow \mathbb{S}^{3} \times[0,1] \backslash W
$$

are homotopy equivalences. Consider the retraction $r_{2}: \mathbb{S}^{3} \times[0,1] \backslash W \rightarrow \mathbb{S}^{3} \backslash K_{2}$. Notice that $r_{2} \circ i_{1}$ induces an isomorphism between the fundamental groups $\pi_{1}\left(\mathbb{S}^{3} \backslash K_{1}\right)$ and $\pi_{1}\left(\mathbb{S}^{3} \backslash K_{2}\right)$. If $K_{1}$ is a trivial knot, then $\pi_{1}\left(\mathbb{S}^{3} \backslash K_{1}\right)=\mathbb{Z}=\pi_{1}\left(\mathbb{S}^{3} \backslash K_{2}\right)$ and thus, $K_{2}$ is also a trivial knot. Hence, we may assume that $K_{1}$ is not a trivial knot. In this case, by Theorem 2.3 , it is enough to show that the isomorphism $\left(r_{2} \circ i_{1}\right) *$ preserves the peripheral structure.

Let $m_{1}$ be a meridian of the knot $K_{1}$. We may suppose that $r_{2} \circ i_{1}\left(m_{1}\right) \subset \partial N\left(K_{2}\right)$ for some solid torus $N\left(K_{2}\right)$ containing the knot $K_{2}$. By the isomorphism condition, $\left[r_{2} \circ i_{1}\left(m_{1}\right)\right]$ is not zero in $\pi_{1}\left(\mathbb{S}^{3} \backslash K_{2}\right)$ because $\left[m_{1}\right] \neq 0$ in $\pi_{1}\left(\mathbb{S}^{3} \backslash K_{1}\right)$. Hence, it suffices to show that $r_{2} \circ i_{1}\left(m_{1}\right)$ is homologically trivial in $N\left(K_{2}\right)$. Otherwise, we have that $\left[r_{2} \circ i_{1}\left(m_{1}\right)\right]$ would be a multiple of $\left[K_{2}\right]$ in $H_{1}\left(N\left(K_{2}\right)\right) \cong \mathbb{Z}$. Since $\left[K_{2}\right]=0$ in $H_{1}\left(\mathbb{S}^{3} \backslash K_{2}\right)$, 
this would imply that $0=\left[r_{2} \circ i_{1}\left(m_{1}\right)\right] \in H_{1}\left(\mathbb{S}^{3} \backslash K_{2}\right)$ ), which is a contradiction (because the homology class is a homotopy invariant). Hence, $r_{2} \circ i_{1}\left(m_{1}\right)$ is a meridian of $K_{2}$.

Now, let $l_{1}$ be a longitude in $\partial N\left(K_{1}\right) \subset \mathbb{S}^{3} \backslash \stackrel{\circ}{N}\left(K_{1}\right)$. Consider $l_{2}=r_{2}\left(i_{1}\left(l_{1}\right)\right)$. We may assume that $l_{2} \subset \partial N\left(K_{2}\right)$ for some solid torus $N\left(K_{2}\right)$. Since $K_{1}$ is not trivial, by Dehn's lemma, the inclusion $i: \partial N\left(K_{1}\right) \rightarrow \mathbb{S}^{3} \backslash \stackrel{\circ}{ }\left(K_{1}\right)$ induces a monomorphism $i_{*}: \pi_{1}\left(\partial N\left(K_{1}\right)\right)=\mathbb{Z} \oplus \mathbb{Z} \rightarrow \pi_{1}\left(\mathbb{S}^{3} \backslash N\left(K_{1}\right)\right)$, where the pair meridian-longitude $\left(m_{1}, l_{1}\right)$ of $K_{1}$ provide generators of $\mathbb{Z} \oplus \mathbb{Z}$. In particular, $l_{1}$ is homologous to the knot $K_{1}$ in $N\left(K_{1}\right)$. Now, we have that $\left\langle r_{2 *}\left(\left[m_{1}\right],\left[l_{1}\right]\right)\right\rangle=\mathbb{Z} \oplus \mathbb{Z} \leq \pi_{1}\left(\mathbb{S}^{3} \backslash \stackrel{\circ}{ }\left(K_{2}\right)\right)$. Since $r_{2 *}\left(m_{1}\right)$ is a meridian, the other class $r_{2 *}\left(l_{1}\right)=\left[r_{2}\left(l_{1}\right)\right]$ is, necessarily, homologous to the knot $K_{2}$.

\section{3. $C^{0}-\mathscr{A}$-EQUIVALENCE AND KNOT TYPE}

In a previous paper [13], we consider the $C^{0}$ - $\mathscr{A}$-classification of analytic map germs $f:\left(\mathbb{R}^{2}, 0\right) \rightarrow\left(\mathbb{R}^{4}, 0\right)$ with isolated singularity. One of the main results of [13] is that two map germs are $C^{0}$ - $\mathscr{A}$-equivalent if their links have the same knot type. In this section, we will prove that the converse is also true, that is, if two map germs are $C^{0}$ - $\mathscr{A}$-equivalent, then their links are equivalent as knots.

Definition 3.1. An analytic map germ $f:\left(\mathbb{R}^{n}, 0\right) \rightarrow\left(\mathbb{R}^{p}, 0\right)$ has isolated singularity at 0 (or isolated instability at 0 ) when for some representative $f: \mathcal{U} \subset \mathbb{R}^{n} \rightarrow \mathcal{V} \subset \mathbb{R}^{p}, f$ is a immersion on $\mathcal{U}-\{0\}$ and a $C^{0}$-embedding on $\mathcal{U}$. In particular, its image $f(\mathcal{U})$ is a topological submanifold with isolated singularity at 0 .

Recall that two $C^{\infty}$ map germs $f, g:\left(\mathbb{R}^{n}, 0\right) \rightarrow\left(\mathbb{R}^{p}, 0\right)$ are said $\mathscr{A}$-equivalent if there exist diffeomorphisms $\phi, \psi$ such that $g=\psi \circ f \circ \phi^{-1}$. If $\phi, \psi$ are homeomorphisms instead of diffeomorphisms, then we say that $f, g$ are $C^{0}-\mathscr{A}$-equivalent.

Given $f:\left(\mathbb{R}^{n}, 0\right) \rightarrow\left(\mathbb{R}^{p}, 0\right)$ and a representative $f: \mathcal{U} \rightarrow \mathcal{V}$ we denote:

$$
\begin{aligned}
& D_{\epsilon}^{p}=\left\{y \in \mathbb{R}^{p}:\|y\|^{2} \leq \epsilon\right\}, \quad S_{\epsilon}^{p-1}=\left\{y \in \mathbb{R}^{p}:\|y\|^{2}=\epsilon\right\}, \\
& \tilde{D}_{\epsilon}^{n}=f^{-1}\left(D_{\epsilon}^{p}\right), \quad \tilde{S}_{\epsilon}^{n-1}=f^{-1}\left(S_{\epsilon}^{p-1}\right) .
\end{aligned}
$$

The cone structure theorem is true for analytic map germs $f:\left(\mathbb{R}^{n}, 0\right) \rightarrow\left(\mathbb{R}^{p}, 0\right)$ with isolated instability. Here we state an adapted version for the case $n=2$ and $p=4$.

Theorem 3.2 (Cone structure theorem). [13, 17] Let $f:\left(\mathbb{R}^{2}, 0\right) \rightarrow\left(\mathbb{R}^{4}, 0\right)$ be an analytic map germ with isolated singularity. There exists $\epsilon_{0}>0$ such that for any $\epsilon$, with $0<\epsilon \leq \epsilon_{0}$ we have:

(1) $\tilde{S}_{\epsilon}^{1}$ is diffeomorphic to the circle $\mathbb{S}^{1}$.

(2) $\left.f\right|_{\tilde{S}_{\epsilon}^{1}}: \tilde{S}_{\epsilon}^{1} \rightarrow S_{\epsilon}^{3}$ is an embedding, whose $\mathscr{A}$-class is independent of $\epsilon$.

(3) $\left.f\right|_{\tilde{D}_{\epsilon}^{2} \backslash\{0\}}: \tilde{D}_{\epsilon}^{2} \backslash\{0\} \rightarrow D_{\epsilon}^{4} \backslash\{0\}$ is $\mathscr{A}$-equivalent to the product map id $\times\left. f\right|_{\tilde{S}_{\epsilon}^{1}}$ : $(0, \epsilon] \times \tilde{S}_{\epsilon}^{1} \rightarrow(0, \epsilon] \times S_{\epsilon}^{3}$.

(4) $\left.f\right|_{\tilde{D}_{\epsilon}^{2}}: \tilde{D}_{\epsilon}^{2} \rightarrow D_{\epsilon}^{4}$ is $C^{0}$-A -equivalent to the cone of $\left.f\right|_{\tilde{S}_{\epsilon}^{1}}: \tilde{S}_{\epsilon}^{1} \rightarrow S_{\epsilon}^{3}$. 
We say that the number $\epsilon_{0}>0$, in Theorem 3.2, is a Milnor-Fukuda radius for $f$. Essentially, the properties (1), (2), (3) and (4) are obtained from the condition of transversality between $f$ and $S_{\epsilon}^{3}$, for all $\epsilon$ such that $0<\epsilon \leq \epsilon_{0}$. Notice that all knots $K_{\epsilon}(f)=f\left(\tilde{S}_{\epsilon}^{1}\right) \subset S_{\epsilon}^{3}$ are equivalent, for $0<\epsilon<\epsilon_{0}$. The class of the knot $K_{\epsilon}(f)$ is denoted by $K(f)$.

Theorem 3.3. Let $f, g:\left(\mathbb{R}^{2}, 0\right) \rightarrow\left(\mathbb{R}^{4}, 0\right)$ be analytic map germs with isolated singularity. Then the following conditions are equivalent:

(1) $f$ is $C^{0}$-A -equivalent to $g$;

(2) $K(f)$ and $K(g)$ are equivalent as knots.

Proof. $(2) \Rightarrow(1)$ : Let $\epsilon<\min \left\{\epsilon_{1}, \epsilon_{2}\right\}$, where $\epsilon_{1}$ is a Milnor-Fukuda radius for $f$ and $\epsilon_{2}$ is a Milnor-Fukuda radius for $g$. Since $K(f)$ and $K(g)$ are equivalent, there exists a homemomorphism $\phi_{\epsilon}: S_{\epsilon}^{3} \rightarrow S_{\epsilon}^{3}$ such that $\phi_{\epsilon}\left(K_{\epsilon}(f)\right)=K_{\epsilon}(g)$. Let $\tilde{S}_{\epsilon}^{1}=f^{-1}\left(S_{\epsilon}^{3}\right)$ and $\tilde{\tilde{S}}_{\epsilon}^{1}=g^{-1}\left(S_{\epsilon}^{3}\right)$ and consider the map $\tilde{\phi}: \tilde{S}_{\epsilon}^{1} \rightarrow \tilde{\tilde{S}}_{\epsilon}^{1}$ given by $\tilde{\phi}_{\epsilon}=\left.g^{-1} \circ \phi_{\epsilon} \circ f\right|_{\tilde{S}_{\epsilon}^{1}}$. Thus, we obtain $\left.g\right|_{\tilde{\tilde{S}}_{\epsilon}^{1}}=\phi_{\epsilon} \circ f \circ \tilde{\phi}_{\epsilon}^{-1}$. Hence, $\left.f\right|_{\tilde{S}_{\epsilon}^{1}}$ and $\left.g\right|_{\tilde{\tilde{S}}_{\epsilon}^{1}}$ are $C^{0}-\mathscr{A}$-equivalent and, by condition (4) in Theorem 3.2 , it follows that $f$ is $C^{0}$ - $\mathscr{A}$-equivalent to $g$.

Proof that $(1) \Rightarrow(2)$ : Let $\psi:\left(\mathbb{R}^{4}, 0\right) \rightarrow\left(\mathbb{R}^{4}, 0\right)$ and $\phi:\left(\mathbb{R}^{2}, 0\right) \rightarrow\left(\mathbb{R}^{2}, 0\right)$ be homeomorphisms such that $g=\psi \circ f \circ \phi^{-1}$. By [13, proposition 2.8], we have that $K(g)$ and $\psi(K(f))$ are cobordant. We will prove that $K(g)$ and $\psi(K(f))$ are in fact invertible cobordant from both ends and thus, $K(f)$ and $K(g)$ are equivalent by Proposition 2.5 .

Let $K(f)=K_{\epsilon_{0}}(f)$, where $\epsilon_{0}$ is a Milnor-Fukuda radius of $f$. Let $D_{\delta}^{4} \subset \psi\left(\stackrel{\circ}{D}_{\epsilon_{0}}^{4}\right)$, where $\delta$ is a Milnor-Fukuda radius of $g$ and let $\epsilon_{1}<\epsilon_{0}$ such that $D_{\epsilon_{1}}^{4} \subset \psi^{-1}\left(\stackrel{\circ}{D}_{\delta}^{4}\right)$. Then,

$$
\phi\left(\tilde{D}_{\epsilon_{1}}^{2}\right)=\phi\left(f^{-1}\left(D_{\epsilon_{1}}^{4}\right)\right) \subset \phi\left(f^{-1}\left(\psi^{-1}\left(\stackrel{\circ}{D}_{\delta}^{4}\right)\right)\right)=g^{-1}\left(\stackrel{\circ}{D}_{\delta}^{4}\right)=\stackrel{\check{D}}{\delta}_{\delta}^{2} .
$$

We know that $\phi\left(\tilde{D}_{\epsilon_{0}}^{2}\right)-\stackrel{\check{D}}{\delta}_{\delta}^{2}$ is homeomorphic to $\mathbb{S}^{1} \times[0,1], \psi\left(D_{\epsilon_{0}}^{4}\right)-\stackrel{\circ}{D}_{\delta}^{4}$ is homeomorphic to $\mathbb{S}^{3} \times[0,1]$ and that $g$ is an embedding from $\phi\left(\tilde{D}_{\epsilon_{0}}^{2}\right)-\tilde{\tilde{D}}_{\delta}^{2}$ to $\psi\left(D_{\epsilon_{0}}^{4}\right)-\stackrel{\circ}{D}_{\delta}^{4}$. So, $W_{12}=$ $g\left(\phi\left(\tilde{D}_{\epsilon_{0}}^{2}\right)-\tilde{D}_{\delta}^{2}\right)$ defines a cobordism between $\psi(K(f))$ and $K(g)=K_{\delta}(g)$. Analogously, $W_{21}=g\left(\tilde{D}_{\delta}^{2}-\phi\left(\tilde{D}_{\epsilon_{1}}^{2}\right)\right)$ gives a cobordism between $K(g)$ and $\psi(K(f))$. The union $W_{12} \cup W_{21}$ is given by $g\left(\phi\left(\tilde{D}_{\epsilon_{0}}^{2}-\tilde{\tilde{D}}_{\epsilon_{1}}^{2}\right)\right)=\psi\left(f\left(\tilde{D}_{\epsilon_{0}}^{2}-\tilde{D}_{\epsilon_{1}}^{2}\right)\right)$, which is trivial by condition (3) of Theorem 3.2. Thus, we have an invertible cobordism from end $\psi(K(f))$. The invertible cobordism from end $K(g)$ is obtained in a similar way.

\section{THE ISOLATED SINGULARITY EXPONENT}

Given an analytic map germ $f:\left(\mathbb{R}^{n}, 0\right) \rightarrow\left(\mathbb{R}^{p}, 0\right)$, we denote by $j^{k} f(0)$ its $k$-jet, that is, its Taylor expansion of order $k$. We recall that $f$ is called $k$-determined (resp. $C^{0}-k$ determined) if for any other map germ $g$ such that $j^{k} f(0)=j^{k} g(0), g$ is $\mathscr{A}$-equivalent to $f$ (resp. $C^{0}-\mathscr{A}$-equivalent to $f$ ). One says that $f$ is finitely determined (resp. $C^{0}$-finitely determined) if it is $k$-determined (resp. $C^{0}-k$-determined) for some $k$.

The isolated singularity condition (in the sense of Definition 3.1) is a generic condition, not only for map germs $f:\left(\mathbb{R}^{2}, 0\right) \rightarrow\left(\mathbb{R}^{4}, 0\right)$, but more generally for map germs 
$f:\left(\mathbb{R}^{n}, 0\right) \rightarrow\left(\mathbb{R}^{p}, 0\right)$, with $p \geq 2 n$. In fact, if such a map germ is finitely determined, then, by the Mather-Gaffney finite determinacy criterion (see [21]), $f$ has isolated instability. But when $p \geq 2 n$, this means that $f$ is a $C^{0}$-embedding in $\mathcal{U}$ and a immersion on $\mathcal{U}-\{0\} \subset \mathbb{R}^{n}$, for some representative $f: \mathcal{U} \rightarrow \mathcal{V}$. In this case, the image of $f$ is a $n$-topological manifold with isolated singularity embedded in $\mathbb{R}^{p}$. In this section, we will use the Lojasiewicz exponent as an invariant which detects the isolated singularity condition of a given analytic map germ $f:\left(\mathbb{R}^{n}, 0\right) \rightarrow\left(\mathbb{R}^{p}, 0\right)$. In other words, the existence of this number guarantees that $M=f(\mathcal{U}) \backslash\{0\}$ is an embedded smooth submanifold. The Lojasiewicz exponent and inequalities are powerful tools to investigate the topology and geometry of analytic maps and analytic sets. For instance, see the works [2, 4, 8, 10].

Let $f:\left(\mathbb{R}^{n}, 0\right) \rightarrow\left(\mathbb{R}^{p}, 0\right)$ be an analytic map germ $(n \leq p)$. For each $i=1, \ldots, p$ there exist analytic functions $\alpha_{i j}:\left(\mathbb{R}^{2 n}, 0\right) \rightarrow \mathbb{R}$, with $j=1, \ldots, n$ such that

$$
f_{i}\left(x^{\prime}\right)-f_{i}(x)=\sum_{j=1}^{n} \alpha_{i j}\left(x, x^{\prime}\right)\left(x_{j}^{\prime}-x_{j}\right) .
$$

Let $\alpha=\left(\alpha_{i j}\right)$ be the $p \times n$ matrix obtained in this way and let $D_{1}, D_{2}, \ldots, D_{r}$ be the minors of order $n$ of $\alpha$. We define the map germ $\tilde{\Delta} f:\left(\mathbb{R}^{2 n}, 0\right) \rightarrow \mathbb{R}^{p+r}$ as

$$
\tilde{\Delta} f\left(x^{\prime}, x\right)=\left(f\left(x^{\prime}\right)-f(x), D_{1}\left(x, x^{\prime}\right), \ldots, D_{r}\left(x, x^{\prime}\right)\right),
$$

where $r=\left(\begin{array}{l}p \\ n\end{array}\right)$.

Let $\tilde{\alpha}_{0}$ be the Lojasiewicz exponent of the map $\tilde{\Delta} f$. That is, $\tilde{\alpha}_{0}$ is the infimum of the numbers $\alpha>0$ which satisty the Lojasiewicz inequality

$$
\left\|\tilde{\Delta} f\left(x^{\prime}, x\right)\right\| \geq C \operatorname{dist}\left(\left(x^{\prime}, x\right), V(\tilde{\Delta} f)\right)^{\alpha},
$$

for some real positive numbers $C, \epsilon$ and for all $\left\|\left(x^{\prime}, x\right)\right\|<\epsilon$, where $V(\tilde{\Delta} f)$ is the zero locus of $\tilde{\Delta} f$.

Remark 4.1. The matrix $\alpha$ is not unique, but it is easy to see that $V(\tilde{\Delta} f)$ does not depend of the choice of $\alpha$. In fact, if $x \neq x^{\prime}$, then $\tilde{\Delta} f\left(x^{\prime}, x\right)=0$ if and only if $f(x)=f\left(x^{\prime}\right)$. Otherwise, if $x=x^{\prime}$, we have that $\alpha(x, x)=D f(x)$ (the Jacobian matrix of $f$ at $x$ ), hence $\tilde{\Delta} f\left(x^{\prime}, x\right)=0$ if and only if $x$ is a non-immersive point of $f$. If $f$ is a $C^{0}$-embedding and an immersion outside of 0 , then $V(\tilde{\Delta} f)=\{0\}$. Moreover, the inequality (11) has the following form:

$$
\left\|\tilde{\Delta} f\left(x^{\prime}, x\right)\right\| \geq C\left\|\left(x^{\prime}, x\right)\right\|^{\alpha} .
$$

Definition 4.2. The number $\tilde{\alpha}_{0}$ is defined as the infimum of those $\alpha>0$ which satisfy the inequality (2) for some real positive numbers $C, \epsilon$ and for all $\left\|\left(x^{\prime}, x\right)\right\|<\epsilon$. The number $\tilde{\alpha}_{0}$ is called the isolated singularity exponent of $f$. We denote it by $\mathcal{L}_{0}(\tilde{\Delta} f)$.

The isolated singularity exponent $\alpha_{0}$ does not depend on the choice of the matrix $\alpha$. In fact, by [14, Proposition 3.1], the ideal $I^{2}(f)$ generated in $\mathscr{E}_{2 n}$ by the components of $\tilde{\Delta} f$ 
is independent of the choice of $\alpha$. Moreover, $\alpha_{0}$ is the Lojasiewicz exponent of the ideal $I^{2}(f)$ with respecto to the maximal ideal in $\mathscr{E}_{2 n}$ and it is well known that the Lojasiewicz exponent is independent of the choice of the system of generators of the ideals.

Remark 4.3. By using the sum norm, we may write the inequality (2) as follows:

$$
\frac{\left\|\tilde{\Delta} f\left(x^{\prime}, x\right)\right\|}{\left\|\left(x^{\prime}, x\right)\right\|^{\alpha}}=\frac{\sum_{j=1}^{p}\left|f_{j}\left(x^{\prime}\right)-f_{j}(x)\right|}{\left\|\left(x^{\prime}, x\right)\right\|^{\alpha}}+\frac{\sum_{i=1}^{r}\left|D_{i}\left(x, x^{\prime}\right)\right|}{\left\|\left(x^{\prime}, x\right)\right\|^{\alpha}} \geq C,
$$

for some $C, \epsilon>0$ and for all $0<\left\|\left(x^{\prime}, x\right)\right\|<\epsilon$. Along the diagonal we have

$$
\frac{\|\tilde{\Delta} f(x, x)\|}{\|(x, x)\|^{\alpha}}=\frac{\sum_{i=1}^{r}\left|D_{i}(x, x)\right|}{\|(x, x)\|^{\alpha}} .
$$

So we need to control only of the term $\sum_{i=1}^{r}\left|D_{i}(x, x)\right| /\|(x, x)\|^{\alpha}$ in order to obtain $\mathcal{L}_{0}(\tilde{\Delta} f)$.

Proposition 4.4. An analytic map germ $f$ has isolated singularity at 0 if and only if $\mathcal{L}_{0}(\tilde{\Delta} f)<\infty$.

Proof. If $f$ has isolated singularity, then $\mathcal{L}_{0}(\tilde{\Delta} f)<\infty$ by construction. Suppose now that $\mathcal{L}_{0}(\tilde{\Delta} f)=\tilde{\alpha}_{0}<\infty$. Let $f(x)=f\left(x^{\prime}\right)$. Then

$$
0=\left\|f\left(x^{\prime}\right)-f(x), D_{1}\left(x, x^{\prime}\right), \ldots, D_{r}\left(x, x^{\prime}\right)\right\| \geq C\left\|\left(x, x^{\prime}\right)\right\|^{\alpha} \geq 0
$$

so $x=x^{\prime}=0$. Hence, $f$ is a $C^{0}$-embedding for some representative. Suppose now that $x$ is a non-immersive point of $f$. Taking $x=x^{\prime}$ in the previous inequality, we have that $0=\left\|D_{1}(x, x), \ldots, D_{r}(x, x)\right\| \geq\|(x, x)\|^{\alpha} \geq 0$. Hence, $x=0$. Thus, $f$ has isolated singularity at 0 .

In [13], we introduced the invariant

$$
\delta(f):=\operatorname{dim}_{\mathbb{R}} \frac{\mathscr{E}_{4}}{I^{2}(f)},
$$

where $\mathscr{E}_{n}$ is the local ring of analytic function germs $\left(\mathbb{R}^{n}, 0\right) \rightarrow \mathbb{R}$. This number detects the finite determinacy property for the map germ $f$, that is, $f$ is finitely determined if and only if $\delta(f)<\infty$. Since isolated singularity does not imply finite determinacy, it may happen that $\delta(f)=\infty$, but $\mathcal{L}_{0}(\tilde{\Delta} f)<\infty$. For instance, consider the map germ $f:\left(\mathbb{R}^{2}, 0\right) \rightarrow\left(\mathbb{R}^{4}, 0\right)$ is given by $f(x, y)=\left(x, y^{2}, y\left(x^{2}+y^{2}\right), 0\right)$.

Remark 4.5. If $f$ has corank 1 at 0 , the isolated singularity exponent of $f$ have a more simple description. After analytic change of coordinates, we may write $f$ in the following form:

$$
f(z, y)=(z, \tilde{f}(z, y))
$$

where $z \in \mathbb{R}^{n-1}, y \in \mathbb{R}$ and $\tilde{f}(z, y)=\left(f_{n}(z, y), \ldots, f_{p}(z, y)\right)$. Then, the matrix $\alpha\left(x, x^{\prime}\right)$ satisfies $\alpha_{i j}\left(x, x^{\prime}\right)=1$ for $i=j \leq n$ and $\alpha_{i j}\left(x, x^{\prime}\right)=0$ for $i \neq j, i, j \leq n$. So, we have that $n-p+1$ minors of $\alpha\left(x, x^{\prime}\right)$ are given by $D_{j}\left(x, x^{\prime}\right)=\alpha_{n j}\left(x, x^{\prime}\right)=\alpha_{n j}(z, y, u, v), j \in$ $\{n \ldots, p\}$. Notice that each $\alpha_{n j}\left(x, x^{\prime}\right)$ can be taken as a cofactor of the others minors. 
Thus, the ideal $I^{2}(f)$ is generated by $\alpha_{n j}\left(x, x^{\prime}\right), j \in\{n, \ldots, p\}$. Now, if we take $z=u$ in $f(z, y)-f(u, v)$ we have

$$
\alpha_{n j}(z, y, z, v)=\frac{f_{j}(z, y)-f_{j}(z, v)}{y-v} .
$$

Hence, from the map germ $\tilde{\Delta}^{1} f:\left(\mathbb{R}^{n+1}, 0\right) \rightarrow \mathbb{R}^{p-n+1}$ defined as

$$
\tilde{\Delta}^{1} f(z, y, u)=\left(\frac{f_{n}(z, u)-f_{n}(z, y)}{u-y}, \ldots, \frac{f_{p}(z, u)-f_{p}(z, y)}{u-y}\right),
$$

we may to obtain $\mathcal{L}_{0}(\tilde{\Delta} f)$ considering the infimum of the numbers $\alpha$ such that

$$
\frac{\left\|\tilde{\Delta}^{1} f(z, y, u)\right\|}{(|z|+|y|+|u|)^{\alpha}} \geq C
$$

for some $\epsilon, C>0$ such that $\|z, y, u\|<\epsilon$.

Let $F:\left(\mathbb{R} \times \mathbb{R}^{n}, 0\right) \rightarrow\left(\mathbb{R} \times \mathbb{R}^{p}, 0\right)$ be an analytic map given by $F(t, x)=\left(t, f_{t}(x)\right)$, such that $f_{t}(0)=0$, for all $t$. We say that $F$ is a 1 -parameter family. We say that a 1-parameter family $F$ is uniformly injective (resp. has uniform isolated singularity) when there exists a representative $F: I \times \mathcal{U} \rightarrow I \times \mathbb{R}^{p}$ such that each $f_{t}, t \in I$, is injective (resp. is injective and immersion outside the origin) on $\mathcal{U}$.

Example 4.6. Let $f_{0}:\left(\mathbb{R}^{2}, 0\right) \rightarrow\left(\mathbb{R}^{4}, 0\right)$ given by $f_{0}(x, y)=\left(x, y^{2}, y^{3}, x^{3} y\right)$. This is a map germ of type $I_{3}$ in the Klotz-Pop-Rieger list of $\mathscr{A}$-simple map-germs (see [1]]). Let us consider the family $F(t,(x, y))=\left(t, f_{t}(x, y)\right)=\left(t,\left(x, y^{2}, y^{3}, x^{3} y+t x^{2} y\right)\right)$ (an unfolding of $f_{0}$ ). This family is uniformly injective. Moreover, for each $t, f_{t}$ has isolated singularity for $\|(x, y)\|<t$. However, since $(-t, 0)$ is a non-immersive point of $f_{t}$, the family $F$ does not have uniform isolated singularity. In order to obtain $\mathcal{L}_{0}\left(\tilde{\Delta} f_{t}\right)$, we consider

$$
\tilde{\Delta}^{1} f_{t}(x, y, u)=\left(u^{2}-y^{2}, u^{3}-y^{3}, x^{3}(y-u)+t x^{2}(u-y), u+y, u^{2}+u y+y^{2}, x^{3}+t x^{2}\right),
$$

hence

$$
\begin{aligned}
\frac{\left\|\tilde{\Delta}_{f_{t}}^{1}(x, y, u)\right\|}{(|x|+|y|+|u|)^{\alpha}} & =\frac{\left|u^{2}-y^{2}\right|}{(|x|+|y|+|u|)^{\alpha}}+\frac{\left|u^{3}-y^{3}\right|}{(|x|+|y|+|u|)^{\alpha}}+\frac{\left|x^{3}(y-u)+t x^{2}(u-y)\right|}{(|x|+|y|+|u|)^{\alpha}} \\
& +\frac{|u+y|}{(|x|+|y|+|u|)^{\alpha}}+\frac{\left|u^{2}+u y+y^{2}\right|}{(|x|+|y|+|u|)^{\alpha}}+\frac{\left|x^{3}+t x^{2}\right|}{(|x|+|y|+|u|)^{\alpha}} .
\end{aligned}
$$

From the last term in the right hand side of the equality, we conclude that $\mathcal{L}_{0}\left(\tilde{\Delta} f_{t}\right)=2$ when $t \neq 0$ and $\mathcal{L}_{0}\left(\tilde{\Delta} f_{0}\right)=3$, when $t=0$.

Example 4.7 (Finitely determined case). Let $F(t, x)=\left(t, f_{t}(x)\right)$ be a family such that $\delta\left(f_{t}\right)<\infty$ and $\mathcal{L}_{0}\left(\tilde{\Delta} f_{t}\right)$ is constant. In particular, each $f_{t}$ is a finitely determined map germ. The following example shows that these conditions are not enough to ensure that $\delta$ is constant along a family:

$$
f_{t}(x, y)=\left(x, y^{2}, y\left(x^{2}+y^{2}\right), y\left(x^{4}+y^{6}+t y^{2}\right)\right) .
$$


We have, for each $t, \delta\left(f_{t}\right)<\infty$. Thus, for each $t, f_{t}$ is a finitely determined map germ and, hence, it has isolated singularity at 0 . Using the Singular software (see [6]), we obtain that $\delta\left(f_{t}\right)=2$, for $t \neq 0$ and $\delta\left(f_{0}\right)=4$. On the other hand, $\mathcal{L}_{0}\left(\tilde{\Delta} f_{t}\right)=2$. Moreover, this family has uniform isolated singularity, because $V\left(\tilde{\Delta} f_{t}\right)=\{0\}$, for all $t$.

Example 4.8. The constancy of the $\delta$-invariant along a family $F(t, x)=\left(t, f_{t}(x)\right)$ implies that it has uniformily isolated singularity (see [13, Lemma 4.2]). From this (and considering the previous example), it seems natural to ask if the constancy of $\mathcal{L}_{0}\left(\tilde{\Delta} f_{t}\right)$ implies uniform isolated singularity. In fact, the question has negative answer as the following example shows:

$$
f_{t}(x, y)=\left(x, y^{2}, y\left(x^{4}+t x y^{2}\right), y\left(y^{4}+t x y^{2}\right)\right) .
$$

We will prove that $\mathcal{L}_{0}\left(\tilde{\Delta} f_{t}\right)=4$, for all $t$. If $t=0$, then

$$
\frac{\left\|\tilde{\Delta} f_{0}(x, y, u)\right\|}{(|x|+|y|+|u|)^{\alpha}}=\left(0, \frac{|y+u|}{(|x|+|y|+|u|)^{\alpha}}, \frac{x^{4}}{(|x|+|y|+|u|)^{\alpha}}, \frac{\left|u^{4}+u^{3} y+u^{2} y^{2} u y^{3}+y^{4}\right|}{(|x|+|y|+|u|)^{\alpha}}\right)
$$

so

$$
\frac{\left\|\tilde{\Delta}_{f_{t}}(x, y,-y)\right\|}{(|x|+2|y|)^{\alpha}}=\left(0,0, \frac{x^{4}}{(|x|+2|y|)^{\alpha}}, \frac{y^{4}}{(|x|+2|y|)^{\alpha}}\right),
$$

which implies that $\mathcal{L}_{0}\left(\tilde{\Delta} f_{0}\right)=4$.

Otherwise, if $t \neq 0$, we have

$$
\frac{\left\|\tilde{\Delta} f_{t}(x, y, u)\right\|}{(|x|+|y|+|u|)^{\alpha}}=\left(0, \frac{|y+u|}{(|x|+|y|+|u|)^{\alpha}}, \frac{x^{4}+t x\left(u^{2}+u y+y^{2}\right)}{(|x|+|y|+|u|)^{\alpha}}, P\right),
$$

with

$$
P=\frac{\left|u^{4}+u^{3} y+u^{2} y^{2} u y^{3}+y^{4}+t x\left(u^{2}+u y+y^{2}\right)\right|}{(|x|+|y|+|u|)^{\alpha}}
$$

In particular,

$$
\frac{\left\|\tilde{\Delta} f_{t}(x, y,-y)\right\|}{(|x|+2|y|)^{\alpha}}=\left(0,0, \frac{\left|x^{4}+t x y^{2}\right|}{(|x|+2|y|)^{\alpha}}, \frac{\left|y^{4}+t x y^{2}\right|}{(|x|+2|y|)^{\alpha}}\right) .
$$

For $y=0$, this gives $\frac{\left\|\tilde{\Delta} f_{t}(x, 0,0)\right\|}{(|x|)^{\alpha}}=\left(0,0, \frac{x^{4}}{(|x|)^{\alpha}}, 0\right)$, hence we must take $\alpha=4$. For $y \neq 0$, the last component vanishes along the curve $x=-\frac{y^{2}}{t}$. It follows that

$$
\frac{\left\|\tilde{\Delta} f_{t}\left(-\frac{y^{2}}{t}, y,-y\right)\right\|}{\left(\frac{y^{2}}{|t|}+2|y|\right)^{\alpha}}=\left(0,0, \frac{y^{4}\left(\frac{y^{4}-t^{4}}{t^{4}}\right)}{\left(\frac{y^{2}}{|t|}+2|y|\right)^{\alpha}}, 0\right) \text {. }
$$

Hence, for each representative $f_{t}$ such that $|y|<t$, we must take $\alpha=4$. Thus, the claim is proved.

However, For $|y|=t$, we have that $\left\|\tilde{\Delta} f_{t}\left(-\frac{y^{2}}{t}, y,-y\right)\right\|=0$. It means that each choice of a representative $f_{t}$ along of this family depends of $t$, i.e., this family does not have uniform isolated singularity. 


\section{5. $C^{0}$-Finite Determinacy}

As we have seen, an analytic map germ $f:\left(\mathbb{R}^{n}, 0\right) \rightarrow\left(\mathbb{R}^{p}, 0\right)(n \leq p)$ with isolated singularity does not have, in general, the finite determinacy property. Inspired on the question in Wall's paper (see [21]), in this section we consider the $C^{0}$-finite determinacy. For analytic function germs $h:\left(\mathbb{R}^{n}, 0\right) \rightarrow \mathbb{R}$, it is well known that isolated singularity implies $C^{0}$-finite determinacy (see Kuo [12]) and there are many papers related to find estimates for the degree of $C^{0}$-finite determinacy (see for instance [3]). In this section, we show that, for real analytic maps germs $f$ from $\mathbb{R}^{2}$ to $\mathbb{R}^{4}$, isolated singularity implies $C^{0}$-finite determinacy (Theorem 5.4). The steps to prove this are the following: For maps $f$ from $\mathbb{R}^{n}$ to $\mathbb{R}^{p}, n \leq p$, we establish a condition for a given unfolding of $f$ to have uniform isolated singularity and a Milnor-Fukuda radius of $f_{t}$ that does not depend of $t$ (Proposition 5.2 and Proposition 5.3). In particular, we obtain that this unfolding, for maps from $\mathbb{R}^{2}$ to $\mathbb{R}^{4}$, has constant knot type.

Consider $\mathscr{E}_{n+1}$ the local ring of analytic function germs $\left(\mathbb{R} \times \mathbb{R}^{n}, 0\right) \rightarrow \mathbb{R}$ and $\mathscr{E}_{n+1}^{p}$ the $\mathscr{E}_{n+1}$-module of analytic map germs $\left(\mathbb{R} \times \mathbb{R}^{n}, 0\right) \rightarrow \mathbb{R}^{p}$. We also denote by $\mathcal{M}_{n}$ the ideal of $\mathscr{E}_{n+1}$ generated by $x_{1}, \ldots, x_{n}$. The following lemma is probably well known, but we include here a proof for completeness. It says that if $g_{t}$ is a 1-parameter family of map germs, such that $g_{0}$ has isolated zero at the origin, then the Lojasiewicz exponent of $g_{0}$ controls the fact that the family has uniform isolated zero at the origin.

Lemma 5.1. Let $G:\left(\mathbb{R} \times \mathbb{R}^{n}, 0\right) \rightarrow\left(\mathbb{R} \times \mathbb{R}^{p}, 0\right)$, $n \leq p$, be a 1-parameter family given by $G(t, x)=\left(t, g_{t}(x)\right)$ and such that $g_{0}^{-1}(0)=\{0\}$. Suppose that $g_{t}-g_{0} \in \mathcal{M}_{n}^{\left[\alpha_{0}\right]+1} \mathscr{E}_{n+1}^{p}$, where $\alpha_{0}$ is a Lojasiewicz exponent of $g_{0}$ and $\left[\alpha_{0}\right]$ is the nearest integer less than $\alpha_{0}$. Then, there exists a representative $G:(-\delta, \delta) \times \mathcal{U} \rightarrow(\delta, \delta) \times \mathbb{R}^{p}$ such that $g_{t}^{-1}(0)=\{0\}$, for all $t \in(-\delta, \delta)$.

Proof. Let $m=\left[\alpha_{0}\right]+1$ and write $g_{t}(x)=g_{0}(x)+t h_{t}(x)$, so that $h_{t} \in \mathcal{M}_{n}^{m} \mathscr{E}_{n+1}^{p}$. We first claim that there exist a representative $G:(-\delta, \delta) \times \mathcal{U} \rightarrow(-\delta, \delta) \times \mathbb{R}^{p}$ and $B>0$ such that $\left\|h_{t}(x)\right\| \leq B\|x\|^{\alpha_{0}}, t \in(-\delta, \delta), x \in \mathcal{U}$.

In fact, $\mathcal{M}_{n}^{m}$ is generated by the monomials $x^{\beta}=x_{1}^{\beta_{1}} \ldots x_{n}^{\beta_{n}}$, with $\beta=\left(\beta_{1}, \ldots, \beta_{n}\right)$ such that $|\beta|=\beta_{1}+\cdots+\beta_{n}=m$. Hence, we can write

$$
h_{t}(x)=\sum_{|\beta|=m} x^{\beta} H_{\beta}(x, t)
$$

for some $H_{\beta} \in \mathscr{E}_{n+1}^{p}$. We fix representatives in $\left(-\delta^{\prime}, \delta^{\prime}\right) \times \mathcal{U}^{\prime}$ and take $0<\delta<\delta^{\prime}$ and $\mathcal{U}=B_{\epsilon}(0)$ such that $\bar{B}_{\epsilon}(0) \subset \mathcal{U}^{\prime}$. If $x=0$, the inequality is immediate. Let $x \neq 0$ and put $r=\|x\|$ and $u=x /\|x\|$. We have

$$
\left\|h_{t}(x)\right\| \leq r^{m} \sum_{|\beta|=m}\left\|u^{\beta} H_{\beta}(r u, t)\right\| \leq B r^{\alpha_{0}}
$$


where $B=\sup \left\{\sum_{|\beta|=m}\left\|u^{\beta} H_{\beta}(r u, t)\right\|: u \in \mathbb{S}^{n-1}, r \in[0, \epsilon], t \in[-\delta, \delta]\right\}$. This concludes the proof of the claim.

Now we can finish the proof of the lemma. Fix a representative $G:(-\delta, \delta) \times \mathcal{U} \rightarrow$ $(-\delta, \delta) \times \mathbb{R}^{p}$ such that $\left\|g_{0}(x)\right\| \geq C\|x\|^{\alpha_{0}}$ and $\left\|h_{t}(x)\right\| \leq B\|x\|^{\alpha_{0}}$, for all $(t, x) \in(-\delta, \delta) \times \mathcal{U}$. Moreover, we can assume that $0<\delta<\frac{C}{2 B}$. For all $(t, x) \in(-\delta, \delta) \times \mathcal{U}$,

$$
\left\|g_{t}(x)\right\|=\left\|g_{0}(x)+t h_{t}(x)\right\| \geq\left\|g_{0}(x)\right\|-|t|\left\|h_{t}(x)\right\| \geq(C-|t| B)\|x\|^{\alpha_{0}}>\frac{C}{2}\|x\|^{\alpha_{0}}
$$

Hence, $g_{t}^{-1}(0)=\{0\}$, for all $t \in(-\delta, \delta)$.

We use Lemma 5.1 to show that if $f_{t}$ is a 1-parameter family of map germs such that $f_{0}$ has isolated singularity, then the isolated singularity exponent $\mathcal{L}_{0}\left(\tilde{\Delta} f_{0}\right)$ controls that $f_{t}$ has uniform isolated singularity.

Proposition 5.2 (Uniform isolated singularity). Let $F:\left(\mathbb{R} \times \mathbb{R}^{n}, 0\right) \rightarrow\left(\mathbb{R} \times \mathbb{R}^{p}, 0\right)$ be a family $F(t, x)=\left(t, f_{t}(x)\right)$ such that $f_{0}$ has isolated singularity. Assume that $f_{t}-f_{0} \in$ $\mathcal{M}_{n}^{k} \mathscr{E}_{n+1}^{p}$, where $k \geq\left[\mathcal{L}_{0}\left(\tilde{\Delta} f_{0}\right)\right]+2$. Then, the family has uniform isolated singularity.

Proof. We consider the map germ $\tilde{\Delta} F:\left(\mathbb{R} \times \mathbb{R}^{2 n}, 0\right) \rightarrow\left(\mathbb{R}^{p+r}, 0\right)$ given by

$$
\tilde{\Delta} F\left(t, x, x^{\prime}\right)=\tilde{\Delta} f_{t}\left(x, x^{\prime}\right)=\left(f_{t}\left(x^{\prime}\right)-f_{t}(x), D_{1 t}\left(x, x^{\prime}\right), \ldots, D_{r t}\left(x, x^{\prime}\right)\right) .
$$

Let $\alpha_{0}=\mathcal{L}_{0}\left(\tilde{\Delta} f_{0}\right)$. We need to show that $\tilde{\Delta} f_{t}-\tilde{\Delta} f_{0} \in \mathcal{M}_{2 n}^{\left[\alpha_{0}\right]+1} \mathscr{E}_{2 n+1}^{p+r}$. By Lemma [5.1, this implies that there exists a representative $\Delta F:(-\delta, \delta) \times \tilde{\mathcal{U}} \rightarrow(-\delta, \delta) \times \mathbb{R}^{p+r}$ such that $V\left(\tilde{\Delta} f_{t}\right)=\Delta f_{t}^{-1}(0)=\{0\}$. Thus, $f_{t}$ is injective and an immersion outside the origin, for all $t \in(-\delta, \delta)$.

Since $k>\left[\alpha_{0}\right]+1$, it is clear that $f_{t}\left(x^{\prime}\right)-f_{t}(x)-\left(f_{0}\left(x^{\prime}\right)-f_{0}(x)\right) \in \mathcal{M}_{2 n}^{\left[\alpha_{0}\right]+1} \mathscr{E}_{2 n+1}^{p}$. So, we only need to show that $D_{i t}-D_{i 0} \in \mathcal{M}_{2 n}^{\left[\alpha_{0}\right]+1}$, for all $i=1,2, \ldots, r$. By construction, $D_{i t}$ are the $n$-minors of a $p \times n$ matrix $\alpha_{t}$ with entries in $\mathscr{E}_{2 n+1}$ such that

$$
f_{t}\left(x^{\prime}\right)-f_{t}(x)=\alpha_{t}\left(x, x^{\prime}\right)\left(x^{\prime}-x\right)
$$

Thus,

$$
f_{t}\left(x^{\prime}\right)-f_{t}(x)-\left(f_{0}\left(x^{\prime}\right)-f_{0}(x)\right)=\left(\alpha_{t}\left(x, x^{\prime}\right)-\alpha_{0}\left(x^{\prime}, x\right)\right)\left(x^{\prime}-x\right)=t \beta_{t}\left(x^{\prime}, x\right)\left(x^{\prime}-x\right),
$$

for some matrix $\beta_{t}$. Since the components of $f_{t}\left(x^{\prime}\right)-f_{t}(x)-\left(f_{0}\left(x^{\prime}\right)-f_{0}(x)\right)$ are in $\mathcal{M}_{2 n}^{k}$, it follows that the entries of $\beta_{t}$ must be in $\mathcal{M}_{2 n}^{k-1}$. Take one of the minors $D_{i t}$. Without loss of generality, we can assume that it is given by the first $n$ rows of $\alpha_{t}$. That is, $D_{i t}=\left[v_{1 t}, \ldots, v_{n t}\right]$, where $v_{j t}$ are the row vectors of $\alpha_{t}$. Then, $v_{j t}=v_{j 0}+t w_{j t}$, where $w_{j t}$ 
are the row vectors of $\beta_{t}$. We have:

$$
\begin{aligned}
D_{i t} & =\left[v_{1 t}, \ldots, v_{n t}\right]=\left[v_{10}+t w_{1 t}, \ldots, v_{n 0}+t w_{n t}\right] \\
& =\left[v_{10}, \ldots, v_{n 0}\right]+t \sum_{j=1}^{n}\left[v_{10}, \ldots, w_{j t}, \ldots, v_{n 0}\right]+\cdots+t^{n}\left[w_{1 t}, \ldots, w_{n t}\right] \\
& =D_{i 0}+t \sum_{j=1}^{n}\left[v_{10}, \ldots, w_{j t}, \ldots, v_{n 0}\right]+\cdots+t^{n}\left[w_{1 t}, \ldots, w_{n t}\right] .
\end{aligned}
$$

Therefore, $D_{i t}-D_{i 0} \in \mathcal{M}_{2 n}^{k-1} \subset \mathcal{M}_{2 n}^{\left[\alpha_{0}\right]+1}$, since $k-1 \geq\left[\alpha_{0}\right]+1$.

The next step is to control that we can take a constant Milnor-Fukuda radius in the family $f_{t}$. We recall that $\epsilon_{0}>0$ is a Milnor-Fukuda radius for a map germ $f$ if $f$ is transverse to all the spheres $S_{\epsilon}^{p-1}$, with $0<\epsilon \leq \epsilon_{0}$. This is equivalent to the fact that $\epsilon$ is a regular value of the function $\|f\|^{2}$, for all $0<\epsilon \leq \epsilon_{0}$. Again this fact will be controlled by the Lojasiewicz exponent of Grad $\|f\|^{2}$, the gradient of $\|f\|^{2}$.

Proposition 5.3 (Uniform regular value). Let $F:\left(\mathbb{R} \times \mathbb{R}^{n}, 0\right) \rightarrow\left(\mathbb{R} \times \mathbb{R}^{p}, 0\right)$ be a family $F(t, x)=\left(t, f_{t}(x)\right)$ such that $f_{0}$ has isolated singularity. Let $\beta_{0}=\mathcal{L}_{0}\left(\operatorname{Grad}\left(\left\|f_{0}\right\|^{2}\right)\right.$ be the Lojasiewicz exponent of $\operatorname{Grad}\left(\left\|f_{0}\right\|^{2}\right)$. Suppose that $f_{t}-f_{0} \in \mathcal{M}_{n}^{k} \mathscr{E}_{n+1}^{p}$, with $k \geq\left[\beta_{0}\right]+1$. Then, there exists $\epsilon_{0}>0$ and a representative $F:(-\delta, \delta) \times \mathcal{U} \rightarrow(-\delta, \delta) \times \mathbb{R}^{p}$ such that $\epsilon$ is a regular value of $\left\|f_{t}\right\|^{2}$, for all $0<\epsilon \leq \epsilon_{0}$ and for all $t \in(-\delta, \delta)$.

Proof. Let $g_{t}=\left\|f_{t}\right\|^{2}$. We first show that 0 is an isolated critical point of $g_{0}$. Suppose this is not true. Then, by the curve selection lemma, there exists a non constant analytic arc $\alpha:(-\delta, \delta) \rightarrow \mathbb{R}^{n}$, with $\alpha(0)=0$, such that $\frac{\partial g}{\partial x_{i}}(\alpha(s))=0$, for all $i=1 \ldots, n$. Thus,

$$
(g \circ \alpha)^{\prime}(s)=\frac{\partial g}{\partial x_{1}}(\alpha(s)) \alpha_{1}^{\prime}(s)+\ldots+\frac{\partial g}{\partial x_{n}}(\alpha(s)) \alpha_{n}^{\prime}(s)=0,
$$

for all $s \in(-\delta, \delta)$. Hence $g \circ \alpha=0$ and $f \circ \alpha=0$, but this gives a contradiction, because $f^{-1}(0)=\{0\}$.

Now, we use Lemma 5.1 for the family $H(t, x)=\left(t, h_{t}(x)\right)$, with $h_{t}=\operatorname{Grad} g_{t}$. We show that $h_{t}-h_{0} \in \mathcal{M}_{n}^{\left[\beta_{0}\right]+1} \mathscr{E}_{n+1}^{p}$. Write $f_{t}=f_{0}+t \beta_{t}$ for some $\beta_{t} \in \mathcal{M}_{n}^{k} \mathscr{E}_{n+1}^{p}$. Take a component of $h_{i t}$ of $h_{t}$. We have:

$$
\begin{aligned}
h_{i t} & =\frac{\partial g_{t}}{\partial x_{i}}=2 \sum_{j=1}^{n} f_{j t} \frac{\partial f_{j t}}{\partial x_{i}} \\
& =2 \sum_{j=1}^{n}\left(f_{j 0}+t \beta_{j t}\right)\left(\frac{\partial f_{j 0}}{\partial x_{i}}+t \frac{\partial \beta_{j t}}{\partial x_{i}}\right) \\
& =h_{i 0}+2 t\left(\sum_{j=1}^{n} f_{j 0} \frac{\partial \beta_{j t}}{\partial x_{i}}+\sum_{j=1}^{n} \beta_{j t} \frac{\partial f_{j 0}}{\partial x_{i}}\right)+2 t^{2} \sum_{j=1}^{n} \beta_{j t} \frac{\partial \beta_{j t}}{\partial x_{i}} .
\end{aligned}
$$

Thus, $h_{t}-h_{0} \in \mathcal{M}_{n}^{k} \mathscr{E}_{n+1}^{p} \subset \mathcal{M}_{n}^{\left[\beta_{0}\right]+1} \mathscr{E}_{n+1}^{p}$, since $k \geq\left[\beta_{0}\right]+1$. By Lemma 5.1, there exists a representative $H:(-\delta, \delta) \times \mathcal{U} \rightarrow(-\delta, \delta) \times \mathbb{R}^{n}$ such that $h_{t}^{-1}(0)=\{0\}$, for all $t \in(-\delta, \delta)$. 
In other words, $g_{t}$ has only a critical point at the origin, for all $t \in(-\delta, \delta)$. In particular, 0 is the only critical value of $g_{t}$, for all $t \in(-\delta, \delta)$.

Finally, we arrive to the main result of this section, which proves that any analytic map germ $f:\left(\mathbb{R}^{2}, 0\right) \rightarrow\left(\mathbb{R}^{4}, 0\right)$ with isolated singularity is $C^{0}$-finitely determined. In fact, the theorem gives an estimate of the degree of $C^{0}$-finite determinacy in terms of the Lojasiewicz exponents.

Theorem 5.4. Let $f:\left(\mathbb{R}^{2}, 0\right) \rightarrow\left(\mathbb{R}^{4}, 0\right)$ be an analytic map germ with isolated singularity. Let $k \geq \max \left\{\left[\mathcal{L}_{0}(\tilde{\Delta} f)\right]+2,\left[\mathcal{L}_{0}\left(\operatorname{Grad}\left(\|f\|^{2}\right)\right)\right]+1\right\}$. Then, $f$ is $C^{0}$-k-determined.

Proof. Given an analytic map $g:\left(\mathbb{R}^{2}, 0\right) \rightarrow\left(\mathbb{R}^{4}, 0\right)$ such that $j^{k} g(0)=j^{k} f(0)$, we write $g=j^{k} f(0)+h$, where $h \in \mathcal{M}_{2}^{k+1} \mathscr{E}_{2}$ and consider the family $G(t, x)=\left(t, g_{t}(x)\right)$ given by $g_{t}(x)=j^{k} f(0)(x)+t h(x)$. Fix a parameter $t_{0} \in \mathbb{R}$, by Proposition 5.2 and Proposition 5.3 , there exists a representative $G:\left(t_{0}-\delta, t_{0}+\delta\right) \times \mathcal{U} \rightarrow\left(t_{0}-\delta, t_{0}+\delta\right) \times \mathbb{R}^{4}$ such that $g_{t}$ is injective and an immersion outside the origin and there exists $\epsilon_{0}>0$ such that for all $0<\epsilon \leq \epsilon_{0}, \epsilon$ is a regular value of each $g_{t}$, for all $t \in\left(t_{0}-\delta, t_{0}+\delta\right)$. This implies that $\epsilon_{0}$ is a Milnor-Fukuda radius for each map $g_{t}$, with $t \in\left(t_{0}-\delta, t_{0}+\delta\right)$ (see Theorem 3.2).

Now, the map $G$ defines an isotopy between the family of knots $K\left(g_{t}\right)=g_{t}(\mathcal{U}) \cap S_{\epsilon_{0}}^{3}$ (see [13, Theorem 4.4]). Hence, by Theorem [3.3, all the map germs $g_{t}$ with $t \in\left(t_{0}-\delta, t_{0}+\delta\right)$ are $C^{0}-\mathscr{A}$-equivalent. Since $\mathbb{R}$ is connected, this implies that all the map germs $g_{t}$ with $t \in \mathbb{R}$ are $C^{0}$ - $\mathscr{A}$-equivalent. In particular, $g$ and $f$ are $C^{0}$ - $\mathscr{A}$-equivalent.

\section{The Double Point EXPOnEnT}

Let $f:\left(\mathbb{R}^{n}, 0\right) \rightarrow\left(\mathbb{R}^{p}, 0\right),(n \leq p)$ be an analytic map germ. Let us consider the map germ $\Delta f:\left(\mathbb{R}^{2 n}, 0\right) \rightarrow \mathbb{R}^{p}, \Delta f\left(x, x^{\prime}\right)=f(x)-f\left(x^{\prime}\right)$. Let $\alpha_{0}$ be the Lojasiewicz exponent of the map $\Delta f$. Then, there exists positive constants $C, \epsilon, \alpha$ such that the following Lojasiewicz inequality holds:

$$
\left\|\Delta f\left(x, x^{\prime}\right)\right\| \geq C \operatorname{dist}\left(\left(x, x^{\prime}\right), V(\Delta f)\right)^{\alpha},
$$

for all $\left\|\left(x, x^{\prime}\right)\right\|<\epsilon$, and $\alpha_{0}$ is the infimum of the exponents $\alpha$ such that the inequality (3) is true. Notice that if $f$ is a injective map, we have that $V(\Delta f))=\{(x, x) ; x \in \mathcal{U}\}$, for some representative. In this case, the inequality (3) looks as follows:

$$
\left\|f(x)-f\left(x^{\prime}\right)\right\| \geq C\left\|x-x^{\prime}\right\|^{\alpha},
$$

for all $\left\|\left(x, x^{\prime}\right)\right\|<\epsilon$.

Definition 6.1. Given an analytic map germ $f:\left(\mathbb{R}^{n}, 0\right) \rightarrow\left(\mathbb{R}^{p}, 0\right),(n \leq p)$, the infimum $\alpha_{0}$ of exponents $\alpha$ such that the inequality (4) holds is called the double point exponent of $f$. We write $\alpha_{0}=\mathcal{L}_{0}(\Delta f)$. .

Remark 6.2. $\mathcal{L}_{0}(\Delta f)<\infty$ if and only if $f$ is injective. 
Example 6.3. Let $\gamma:(\mathbb{R}, 0) \rightarrow\left(\mathbb{R}^{3}, 0\right)$ be a germ of an analytic arc with isolated singularity, where $\gamma$ is injective. Let $f:\left(\mathbb{R}^{2}, 0\right) \rightarrow\left(\mathbb{R}^{4}, 0\right)$ be the constant unfolding $f(x, y)=$ $(x, \gamma(y))$. We have that $\tilde{\Delta} f(x, y, u)=\left(0, \gamma(u)-\gamma(y), \Delta_{1} \gamma(y, u)\right)$, where $\Delta_{1} \gamma(y, u)=$ $(\gamma(u)-\gamma(y)) /(u-y)$. If $\tilde{\Delta}_{f}(x, y, u)=0$ then $u=y$ and hence, $0=\Delta_{1} \gamma(y, y)=\gamma^{\prime}(y)$. Therefore, $y=u=0$. It means that $V(\tilde{\Delta} f)=\{(x, 0,0)\}$, i.e., the non-immersive locus of $f$ is the $x$-axes. By Proposition $4.4, \mathcal{L}_{0}\left(\tilde{\Delta}_{f}\right)=\infty$.

On the other hand, if $\gamma(y)$ contains at least a monomial of odd degree $y^{2 m+1}$, we have $\mathcal{L}_{0}\left(\Delta_{f}\right)=2 l$, where $2 l+1$ is the smallest odd exponent of a monomial in $\gamma(y)$.

Lemma 6.4. Let $f:\left(\mathbb{R}^{n}, 0\right) \rightarrow\left(\mathbb{R}^{p}, 0\right),(n \leq p)$ be analytic and injective. Then $\alpha_{0}=$ $\mathcal{L}_{0}(\Delta f) \geq 1$. In particular $f^{-1}$ is a $\frac{1}{\alpha_{0}}$ continuous Hölder map on the image.

Proof. Since $f$ is analytic, it is locally Lipschitz near 0 . Thus, there exist constants $\tilde{C}, \delta_{1}>0$ such that

$$
\frac{\left\|f(x)-f\left(x^{\prime}\right)\right\|}{\left\|x-x^{\prime}\right\|} \leq \tilde{C}
$$

for all $0<\left\|\left(x, x^{\prime}\right)\right\|<\delta_{1}$. On the other hand, there exist positive constants $C, \delta_{2}, \alpha_{0}$ such that

$$
\frac{\left\|f(x)-f\left(x^{\prime}\right)\right\|}{\left\|x-x^{\prime}\right\|^{\alpha_{0}}}>C
$$

for all $\left\|\left(x, x^{\prime}\right)\right\|<\delta_{2}$. Let $\epsilon=\min \left\{\delta_{1}, \delta_{2}\right\}$. If $\alpha_{0}<1$, we can write

$$
\frac{\left\|f(x)-f\left(x^{\prime}\right)\right\|}{\left\|x-x^{\prime}\right\|^{\alpha_{0}}}=\frac{\left\|f(x)-f\left(x^{\prime}\right)\right\|}{\left\|x-x^{\prime}\right\|}\left\|x-x^{\prime}\right\|^{1-\alpha_{0}} \rightarrow 0,
$$

when $\left\|\left(x, x^{\prime}\right)\right\| \rightarrow 0$. But this a contradiction with (5) and thus, we must have $\alpha_{0} \geq 1$.

Let $f, g:\left(\mathbb{R}^{n}, 0\right) \rightarrow\left(\mathbb{R}^{p}, 0\right)$ be analytic map germs. Then $f$ and $g$ are called bi-Lipschitz equivalent if there exist bi-Lipschitz homeomorphisms $\phi:\left(\mathbb{R}^{n}, 0\right) \rightarrow\left(\mathbb{R}^{n}, 0\right)$ and $\psi:\left(\mathbb{R}^{p}, 0\right) \rightarrow$ $\left(\mathbb{R}^{p}, 0\right)$ such that $g=\psi \circ f \circ \phi^{-1}$. In the next lemma, we show that $\mathcal{L}_{0}(\Delta f)$ is a bi-Lipschitz invariant.

Lemma 6.5. If $f$ and $g$ are bi-Lipschitz equivalent then, $\mathcal{L}_{0}(\Delta f)=\mathcal{L}_{0}(\Delta g)$.

Proof. First, it is immediate that $\mathcal{L}_{0}(\Delta f)=\infty$ if and only if $\mathcal{L}_{0}(\Delta g)=\infty$, so we can assume both numbers are finite. Let $\alpha_{0}=\mathcal{L}_{0}(\Delta f)<\infty$ and assume that $g=f \circ \phi$, where $\phi:\left(\mathbb{R}^{n}, 0\right) \rightarrow\left(\mathbb{R}^{n}, 0\right)$ is a bi-Lipschitz homeomorphism. We have:

$$
\left\|g(x)-g\left(x^{\prime}\right)\right\|=\left\|f \circ \phi(x)-f \circ \phi\left(x^{\prime}\right)\right\| \geq C\left\|\phi(x)-\phi\left(x^{\prime}\right)\right\|^{\alpha_{0}} \geq C \tilde{C}\left\|x-x^{\prime}\right\|^{\alpha_{0}},
$$

for some positive constants $C, \tilde{C}$ and for all $\left\|\left(x, x^{\prime}\right)\right\|<\epsilon$. Thus, $\mathcal{L}_{0}(\Delta g) \leq \alpha_{0}=\mathcal{L}_{0}(\Delta f)$. Using the same argument for $f=g \circ \phi^{-1}$, we obtain $\mathcal{L}_{0}(\Delta g) \geq \mathcal{L}_{0}(\Delta f)$. Hence, $\mathcal{L}_{0}(\Delta g)=$ $\mathcal{L}_{0}(\Delta f)$.

On the other hand, assume that $g=\psi \circ f$, where $\psi:\left(\mathbb{R}^{p}, 0\right) \rightarrow\left(\mathbb{R}^{p}, 0\right)$ is a bi-Lipschitz homeomorphism. We have:

$$
\left\|g(x)-g\left(x^{\prime}\right)\right\|=\left\|\psi(f(x))-\psi\left(f\left(x^{\prime}\right)\right)\right\| \geq D\left\|f(x)-f\left(x^{\prime}\right)\right\| \geq D \tilde{D}\left\|x-x^{\prime}\right\|^{\alpha_{0}},
$$


for some positive constants $D, \tilde{D}$ and for all $\left\|\left(x, x^{\prime}\right)\right\|<\epsilon$. Thus, $\mathcal{L}_{0}(\Delta g) \leq \mathcal{L}_{0}(\Delta f)$ and, in a similar way (by considering $\psi^{-1} \circ g$ ), we obtain $\mathcal{L}_{0}(\Delta g) \geq \mathcal{L}_{0}(\Delta f)$, so $\mathcal{L}_{0}(\Delta g)=\mathcal{L}_{0}(\Delta f)$.

Now, if $g=\psi \circ f \circ \phi^{-1}$, then $\mathcal{L}_{0}(\Delta g)=\mathcal{L}_{0}(\Delta f \circ \phi)=\mathcal{L}_{0}(\Delta f)$.

The main result of this section is that any Lipschitz embedding is a smooth embedding.

Theorem 6.6. Let $f:\left(\mathbb{R}^{n}, 0\right) \rightarrow\left(\mathbb{R}^{p}, 0\right), n \leq p$, be analytic and injective. Then, the following assertions are equivalent:

(1) $f$ is a bi-Lipschitz map on the image;

(2) $\mathcal{L}_{0}(\Delta f)=1$;

(3) $f$ is a smooth embedding.

Proof. Notice that $(2) \Leftrightarrow(1)$ is immediate from the definition of $\mathcal{L}_{0}(\Delta f)$ and that $(3) \Rightarrow$ (1) is also clear. So, we only have to prove $(2) \Rightarrow(3)$. Assume that 0 is a non-immersive point of $f$. Let $0<r \leq n$ be the corank of $f$ at 0 . After $\mathscr{A}$-equivalence, we may write

$$
f(z, y)=(z, \tilde{f}(z, y)), z \in \mathbb{R}^{n-r}, y \in \mathbb{R}^{r},
$$

where $\tilde{f}(z, y)=\left(f_{n-r+1}(z, y), \ldots f_{p}(z, y)\right)$ and $f_{n+r+1}, \ldots, f_{p} \in \mathcal{M}_{n}^{2}$.

For each $f_{i}$, with $i=n-r+1, \ldots, p$, we write

$$
f_{i}(z, u)-f_{i}(z, y)=\sum_{j=1}^{r} \alpha_{i j}(z, y, u)\left(y_{j}-u_{j}\right)
$$

for some $\alpha_{i j} \in \mathcal{M}_{r+n}$. By using matrix notation $\alpha=\left(\alpha_{i j}\right)$, we have

$$
\tilde{f}(z, u)-\tilde{f}(z, y)=\alpha(z, y, u)(u-y),
$$

with $\alpha(0)=0$. Let $\|\alpha\|$ be the matrix norm of $\alpha$. If $u \neq y$, then:

$$
\frac{\|\tilde{f}(z, u)-\tilde{f}(z, y)\|}{\|u-y\|}=\frac{\|\alpha(z, y, u)(u-y)\|}{\|u-y\|} \leq \frac{\|\alpha(z, y, u)\|\|(u-y)\|}{\|u-y\|}=\|\alpha(z, y, u)\| \rightarrow 0,
$$

when $\|(z, y, u)\| \rightarrow 0$.

On the other hand, assume that $\mathcal{L}_{0}(\Delta f)=1$. If $(z, y) \neq\left(z^{\prime}, u\right)$, then

$$
\frac{\left\|z^{\prime}-z\right\|+\left\|\tilde{f}\left(z^{\prime}, u\right)-\tilde{f}(z, y)\right\|}{\left\|z^{\prime}-z\right\|+\|u-y\|} \geq C
$$

for some $C>0$ and for all $\left\|\left(z, y, z^{\prime}, u\right)\right\|<\epsilon$. In particular, for $z=z^{\prime}$ and $y \neq u$,

$$
\frac{\|\tilde{f}(z, u)-\tilde{f}(z, y)\|}{\|u-y\|} \geq C
$$

for all $\|(z, y, u)\|<\epsilon$. But this gives a contradiction with (7).

Corollary 6.7. Let $X \subset \mathbb{R}^{p}$ be an analytic subset. If $X$ locally parametrized at $x_{0}$ as the image of an analytic map which is bi-Lipschitz onto its image, then $X$ is smooth at $x_{0}$. 
In the complex case, Birbrair, Lê, Fernandes and Sampaio show in [1] that if a complex algebraic set $X \subset \mathbb{C}^{n}$ is Lipschitz regular at a point $x_{0} \in X$, then $X$ is smooth at $x_{0}$. This is false in the real case, for instance, the surface in $\mathbb{R}^{3}$ given by $x^{3}+y^{3}=z^{3}$ is Lipschitz regular at the origin, but it is not smooth (see [16]). Thus, our corollary can be seen as a weaker real version of that theorem.

Remark 6.8. The same argument of the proof of Theorem 6.6 works is $f$ not analytic, but is $C^{1}$. In that case, the exponent $\mathcal{L}_{0}(\Delta f)$ is not defined, but one can show that if $f$ is a $C^{1}$ map which is bi-Lipschitz onto its image, then $f$ is a $C^{1}$-embedding.

Acknowledgements. We would like to thank Osamu Saeki and Carles Bivià-Ausina by the extremely useful suggestions.

\section{REFERENCES}

[1] L. Birbrair, A. Fernandes, Lê, D.T. and J.E. Sampaio, Lipschitz regular complex algebraic sets are smooth. Proc. Amer. Math. Soc. 144 (2016), no. 3, 983-987.

[2] C. Bivià-Ausina, Injectivity of real polynomial maps and Lojasiewicz exponents at infinity, Math. Z. 257 (2007), no. 4, 745-767.

[3] C. Bivià-Ausina, A method to estimate the degree of $C^{0}$-sufficiency of analytic functions. Experiment. Math. 11 (2002), no. 1, 81-85.

[4] J. Bochnak and J.J. Risler, Sur les exposants de Lojasiewicz. Comment. Math. Helv. 50 (1975), no. $4,493-507$.

[5] H. Brodersen, G. Ishikawa and L. Wilson, A criterion for finite topological determinacy of map-germs. Proc. London Math. Soc. (3) 74 (1997), no. 3, 662-700.

[6] W. Decker, G.-M. Greuel, G. Pfister and H. Schönemann, Singular 4-1-1 — A computer algebra system for polynomial computations. http://www.singular.uni-kl.de (2018).

[7] T. Fukuda, Local Topological Properties of Differentiable Mappings I, Invent. Math. 65 no. 2 (1981/82) 227-250.

[8] J. Gwodziewicz, The Lojasiewicz exponent of an analytic function at an isolated zero. Comment. Math. Helv. 74 (1999), no. 3, 364-375.

[9] C. McA Gordan and J. Luecke, Knots are determined by their complements, J. Amer. Math. Soc. 2 (1989), no. 2, 371-415.

[10] Z. Jelonek, On the Lojasiewicz exponent, Hokkaido Math. J. 35 (2006), no. 2, 471-485.

[11] C. Klotz, O. Pop and J.H. Rieger, Real double-points of deformations of $\mathscr{A}$-simple map-germs from $\mathbb{R}^{n}$ to $\mathbb{R}^{2 n}$. Math. Proc. Cambridge Philos. Soc. 142 (2007), no. 2, 341-363.

[12] T.C. Kuo, On $C^{0}$-sufficiency of jets of potential functions. Topology 8 (1969), 167-171.

[13] R. Mendes and J. J. Nuño-Ballesteros, Knots and the topology of singular surfaces in $\mathbb{R}^{4}$. Real and complex singularities, 229-239, Contemp. Math., 675, Amer. Math. Soc., Providence, RI, 2016.

[14] D. Mond, Some remarks on the geometry and classification of germs of maps from surfaces to 3-space. Topology 26 (1987), no. 3, 361-383.

[15] C.D. Papakyriakopoulos, On Dehn's Lemma and the Asphericity of Knots, Proc. Natl. Acad. Sci. USA 43 (1) (1957), 169-172.

[16] J.E. Sampaio, Regularidade Lipschitz, invariância da multiplicidade e a geometria dos cones tangentes de conjuntos analíticos. PhD thesis, Universidade Federal do Ceará, 2015. 
[17] L.C. Siebenmann, Pseudo annuli and invertible cobordism. Arch. der Math. 19 (1968), 528-535.

[18] J.R. Stallings, J. R. On infinite processes leading to differentiability in the complement of a point. 1965 Differential and Combinatorial Topology (A Symposium in Honor of Marston Morse) pp. 245254 Princeton Univ. Press, Princeton, N.J.

[19] D.W. Sumners, Invertible Knot Cobordisms. Com. Math. Helv. 46 (1975), 240-256.

[20] F. Waldhausen, On irreducible 3-manifolds which are sufficiently large, Annals of Mathematics $\mathbf{8 7}$ (1) (1067), 56-88.

[21] C.T.C. Wall, Finite determinacy of smooth map germs, Bull. London. Math. Soc. 13 (1981), 481-539.

Departament de Matemàtiques, Universitat de València, Campus de Burjassot, 46100 BuRJassot, Spain

E-mail address: Juan. Nuno@uv.es

Instituto de Ciências exatas e da natureza, Universidade de IntegraÇão Internacional da Lusofonia Afro-Brasileira (UnilaB), Campus dos Palmares, Cep. 62785-000. AcarapeCe, Brasil

E-mail address: rodrigomendes@unilab.edu.br 